\title{
Evaluación del Potencial Mutagénico de Piroxicam, Meloxicam y Precursores mediante el Ensayo de Micronúcleos in vivo
}

Javier H. Gris, Martín A. Dragonetti, Beatriz M. Fernández y Susana M. Sicardi

Universidad de Buenos Aires, Facultad de Farmacia y Bioquímica, Cátedra de Química Medicinal, Junín 956, (1113) Buenos Aires-Argentina (e-mail: javierg@ffyb.uba.ar)

\begin{abstract}
Resumen
El potencial mutagénico del piroxicam (1), 2-aminopiridina (2), meloxicam (3) y 2-amino-5-metil tiazol (4) ha sido evaluado mediante el ensayo de micronúcleos in vivo. Se administraron 4 niveles de dosis para cada compuesto y las células de la médula ósea fueron examinadas a las 24 horas posteriores a la administración. En el caso del producto de degradación del piroxicam, solamente ha sido evaluado a $0.1,0.3,1.0$ y $5.0 \mathrm{mg} / \mathrm{kg}$, ya que a $50 \mathrm{mg} / \mathrm{kg}$ todos los animales mueren. Los resultados muestran que las dosis ensayadas no inducen un incremento estadísticamente significativo en la frecuencia de eritrocitos policromáticos micronucleados. La anilina (5) ha sido usada como control positivo, mostrando una respuesta favorable en la inducción de micronúcleos a la dosis de $50 \mathrm{mg} / \mathrm{kg}$ o superiores, mientras que a dosis inferiores no induce micronúcleos. La relación estructura-actividad entre piroxicam y aniline no pudo ser establecida.
\end{abstract}

\section{Evaluation of the Mutagenic Potential of Piroxicam, Meloxicam and their Precursors using in vivo Micronucleus Test}

\begin{abstract}
Mutagenic potential of piroxicam (1), 2-aminopyridine (2), meloxicam (3) and 2-amino-5-methylthiazole (4) has been evaluated using in vivo micronucleus test. Four dose levels for each compound were used and bone marrow cells were examined 24 hours after the administration. In the case of the degradation product of piroxicam (2) only $0.1,0.3,1.0$ and $5.0 \mathrm{mg} / \mathrm{kg}$ were evaluated, because at $50 \mathrm{mg} / \mathrm{kg}$ all animals died. The results show that doses applied do not induce a statistical significant increase of the frequency of micronucleated polychromatic erythrocytes. Aniline (5) has been used as positive mutagen reference, showing a positive response to the micronucleous induction at doses of $50 \mathrm{mg} / \mathrm{kg}$ or superior while no induction occur with lower doses. The structural-mutagenic activity relationship between piroxicam and aniline could not been established.
\end{abstract}

Keywords: piroxicam, meloxicam, mutagenic potential, 2-aminopyridine, aniline 


\section{INTRODUCCIÓN}

El piroxicam (4-hidroxi-2-metil-N-(2-piridinil)-2H-1,2-benzotiazina-3-carboxamida 1,1-dióxido) y el meloxicam (4-hidroxi-2-metil-N-(5-metil-2-tiazolil)-2H-1,2-benzotiazina-3-carboxamida 1,1-dióxido), son antiinflamatorios no esteroides (AINEs) pertencientes a la familia de los llamados "oxicanes", las estrructuras se pueden apreciar en la figura 1. Ambos actúan por inhibición de la ciclooxigenasa (COX), destacándose entre los AINEs por su larga duración de acción. El piroxicam (Lombardino et al., 1971) es el primer compuesto comercializado de esta familia, desarrollado a principios de la década del 70, caracterizándose por ser un inhibidor no selectivo, ya que inhibe a ambas isoformas (COX-1 y COX-2) de la COX (Griswold y Adams, 1999), mientras que el meloxicam, comercializado en 1996 aunque actúa sobre ambas isoformas es unas 4 veces más selectivo hacia la COX-2 (Bianchi y Panerai, 2002)<smiles>CN1C(C(=O)Nc2ccccn2)=C(O)c2ccccc2S1(=O)=O</smiles>

Piroxicam (1)<smiles>Nc1ccccn1</smiles>

2-amino piridina (2)<smiles>Cc1cnc(NC(=O)C2=C(O)c3ccccc3S(=O)(=O)N2C)s1</smiles>

meloxicam (3)<smiles>Cc1cnc(N)s1</smiles>

2-amino-5-metil tiazol<smiles>Nc1ccccc1</smiles>

anilina (5)

Fig. 1: Estructura química de los compuestos ensayados

Varios compuestos con elevada selectividad hacia la COX-2 han sido desarrollados en los últimos años, con un efecto secundario sobre el tracto gastrointestinal mucho más reducido; como lo han demostrado los comercializados meloxicam cuatro veces más selectivo (4x), nimesulida 8 veces más selectivo (8x) y celecoxib 16 veces más selectivo (16x) entre otros (Lazer et al., 1997; Praveen et al., 2004).

De la familia de los oxicanes sólo el meloxicam es algo más selectivo y estudios clínicos sobre su farmacocinética en 78 voluntarios han demostrado que tiene una mayor vida media que el piroxicam siendo suficiente una sola toma diaria para el tratamiento de la osteoartritis y artritis reumatoidea (Lipscomb et al., 1998; Dequeker et al., 1998). En los últimos años, la comercialización de meloxicam se ha incrementado, tanto en Argentina como a nivel mundial por la seguridad de su perfil terapéutico pero sobre todo debido al retiro del mercado de otros inhibidores selectivos de la COX-2 pertencientes a la familia de los diarilheterociclos (llamados Coxibs). Los coxib o diarilheterociclos, entre los cuales se encuentran el celecoxib, rofecoxib, valdecoxib, parecoxib, etoricoxib y el más reciente de todos el lumiracoxib (Blobaum y Marnett, 2007). A partir de su descubrimiento a finales de la década del noventa, rápidamente acapararon el mercado farmacéutico y se constituyeron en los fármacos de elección para el tratamiento de la artritis y la artrosis, especialmente en personas mayores. Sin embargo, en septiembre de 2004 el laboratorio Merck Sharp \& Dome retiró del mercado mundial el producto rofecoxib, motivados en la necesidad de verificar si el fármaco contribuía al incremento de posibilidades de infarto del miocardio o accidente cerebrovascular (Tejer y Doval, 2004). 
Teniendo en cuenta la escasez de publicaciones relacionadas con nuevos derivados de oxicanes, y con el conocimiento de la importancia que tiene la búsqueda de nuevos análogos con mayor selectividad hacia la COX-2; este grupo de trabajo intenta, previo a nuevas síntesis, constatar que estos compuestos o sus precursores no resulten mutagénicos y/o carcinogénicos. Bajo esta hipótesis se ha elegido el Ensayo de Micronúcleos in vivo iniciado por Schmid (Schmid, 1975), basándose en los siguientes principios: cuando un cromosoma se rompe, si la migración del fragmento acéntrico se retrasa entre los polos y se transforma en un núcleo más pequeño que el núcleo principal, es llamado Micronúcleo. Éste es formado durante la anafase/telofase y de este modo la división celular es requerida para que el daño cromosómico / genómico sea expresado como micronúcleo. Un aspecto fundamental para el ensayo es la cinética de división. Un micronúcleo normalmente debe contener un cromosoma completo o un fragmento cromosómico acéntrico.

En investigaciones previas (Sicardi y Martiarena, 1986a; Sicardi y Martiarena, 1986b, Sicardi et al., 1991; Sicardi y Ferrato, 1995) se evidenció la acción mutagénica positiva de la anilina, con mejoras en el Protocolo Internacional del ensayo; por esta razón en esta investigación será utilizada como patrón de referencia. Por otra parte, la semejanza de la 2-amino piridina con la anilina, justifica la necesidad de probar el nitrógeno heterocíclico de la molécula como posible agente mutagénico; sobre esta base, los dos bioisósteros serán probados a una misma dosis. (50 mg/kg). La 2-amino piridina y el 2-amino-5-metil tiazol fueron incluidos pues pueden, como precursores de síntesis, encontrarse como impurezas y además pueden generar, luego de un año de conservación productos de degradación.

\section{MATERIALES Y MÉTODOS}

\section{Animales}

Ratones S.J.L. Swiss machos y hembras con una edad comprendida entre 8 y 12 semanas de vida, contando con un peso de 20-25 gramos al inicio del período de tratamiento. Los mismos fueron adquiridos al Bioterio de la Facultad de Farmacia y Bioquímica, de la Universidad de Buenos Aires. Los mismos fueron criados en nuestro laboratorio y mantenidos a $20-25{ }^{\circ} \mathrm{C}$, con libre acceso al agua y alimentos (Nutrimentos S.A., dieta 3), respetando el ciclo de 12 horas luz y 12 horas oscuridad; hasta el tiempo de experimentación.

\section{Sustancias de ensayo}

Piroxicam, 2-aminopiridina, meloxicam, 2-amino-5-metil tiazol y anilina fueron obtenidos por vía comercial y purificadas por cristalización o destilación respectivamente. Todos los compuestos fueron analizados por cromatografía en capa delgada (TLC) para garantizar su pureza.

\section{Ensayo de micronúcleos in vivo}

En el presente estudio para cada dosis fueron utilizados 8 ratones Swiss cuatro de cada sexo, exceptuando el grupo tratado con anilina para el cual se utilizaron 10 ratones, cinco por sexo.

De cada lote de animales, un grupo fue usado como control (tabla 1, dosis 0) inyectándoles únicamente el vehículo -una solución acuosa de propilenglicol $(0.15 \mathrm{ml} / \mathrm{ml})$-, vehículo en el cual las sustancias de ensayo fueron disueltas o suspendidas a la concentración deseada e inmediatamente antes de su administración. La selección de la dosis máxima para este ensayo se basó en la posibilidad práctica de obtener suspensiones homogéneas para la administración; mientras que las dosis bajas se seleccionaron por sugerencias de protocolos previos (Mc Gregor et al., 1987; Schmid, 1975; Hayashi et al., 1984; Sicardi y Martiarena, 1986a; Sicardi et al., 1991), por reducción de la dosis máxima al $1 \frac{1}{2}, 1 / 4$, etc.

Las soluciones y suspensiones fueron administradas por vía intraperitoneal una sola vez y a las 24 horas posteriores a la administración se llevó a cabo la eutanasia de los animales por dislocación cervical. Para cada ratón, ambos fémures fueron removidos, preparándose en portaobjetos, extendidos de la médula ósea, tal como fue descrito en publicaciones anteriores (Sicardi et al, 1991). La presencia de micronúcleos (MN) fue analizada en 1000 eritrocitos policromáticos (EPC) para cada 
animal y dosis en portaobjetos codificados y el número de eritrocitos policromáticos micronucleados (EPCMN) fue determinada para cada animal.

\section{Análisis estadístico}

Los datos fueron analizados usando Instant 2 GraphPad Inc. Sofware. El análisis de Bartlett fue aplicado para determinar la homogeneidad de varianza entre los grupos. El método de análisis de varianza de un solo factor -"one way- ANOVA test" fue elegido para detectar diferencias significativas en la frecuencia de micronúcleos entre animales tratados y controles. En caso de existir diferencias, el análisis de Newman-Keuls permite individualizar las mismas. En todos los casos, una p menor a 0.05 es considerada estadísticamente significativa.

\section{RESULTADOS y DISCUSIÓN}

La presencia de micronúcleos (MN) fue analizada en 1000 eritrocitos policromáticos (EPC) para cada animal y dosis en portaobjetos codificados y el número de eritrocitos policromáticos micronucleados (EPCMN) fue determinada para cada animal. Además se calculó para cada grupo de tratamiento el promedio de EPCMN y la desviación estándar (DE), los datos se muestran en la tabla 1. La columna 4 describe el promedio de los datos individuales y la desviación standard media (DEM). La relación de eritrocitos policromáticos a normocromáticos (EPC/ENC) fue determinada en 200 eritrocitos totales en cada preparación.

En el recuento de micronúcleos de los grupos de tratamiento no se observaron diferencias significativas entre animales machos y hembras, por lo cual los datos fueron analizados en conjunto $n=10$ para la anilina y $n=8$ para el resto de los compuestos ensayados.

Los datos muestran que en los grupos de tratamiento administrados con piroxicam, meloxicam, 2amino piridina y 2-amino-5-metil tiazol no se observaron diferencias estadísticamente significativas en la frecuencia de EPCMN al compararse con los correspondientes animales controles por lo que los resultados se consideran negativos y los compuestos como no genotóxicos bajo las condiciones del ensayo. En los animales administrados con la anilina se observaron incrementos significativos en la frecuencia de EPCMN por lo que se considera como un agente genotóxico. Los resultados positivos obtenidos coinciden con otros, publicados previamente (Sicardi y Martiarena 1986a; Sicardi et al., 1991; Ashby et al., 1991).

El recuento para relacionar eritrocitos policromáticos micronucleados a normocromáticos, se mantuvo en un rango de 0.9:1 a 1.4:1 por lo cual no se incluyeron en la tabla 1 y estos resultados indican que los compuestos ensayados, a las dosis administradas no afectan la proliferación celular de la médula ósea. Los datos ausentes se deben a dificultades técnicas en el extendido.

En el caso del piroxicam y meloxicam los resultados negativos, coinciden con los publicados previamente; por ejemplo en las fichas técnicas del meloxicam cuyo nombre comercial es Mobic ${ }^{\circledR}$ y piroxicam cuyo nombre comercial es Feldene ${ }^{\circledR}$ (Approved Drug label), se establece que no provocan efectos genotóxicos en los estudios in vitro e in vivo realizados durante su desarrollo preclínico, como tampoco presentan efectos carcinogénicos en los estudios realizados en animales de experimentación. No ha sido posible analizar a niveles de dosis equivalentes, la influencia del nitrógeno en el anillo heterocíclico de la 2-amino piridina respecto a la estructura de la anilina ambas bioisósteras- debido a que en esta última el efecto mutagénico aparece a la dosis de 50 $\mathrm{mg} / \mathrm{kg}$, mientras que a esta dosis la letalidad es del $100 \%$ en los animales tratados con la 2-amino piridina; así como tampoco se realizó la comparación de los resultados cuantitativos de los compuestos en estudio en referencia a la anilina.

Las dosis máximas de piroxicam y meloxicam utilizadas en este ensayo (50 mg/kg), son superiores a las dosis máximas terapéuticas recomendadas en humanos para ambos productos (20 mg/día y 15 $\mathrm{mg} /$ día para piroxicam y meloxicam respectivamente) Teniendo en cuenta la dosis máxima terapéutica recomendada indicada arriba y considerando un paciente de $60 \mathrm{Kg}$ la dosis en humanos es de $0.33 \mathrm{mg} / \mathrm{kg}$ para el piroxicam y $0.25 \mathrm{mg} / \mathrm{kg}$ para el meloxicam, la dosis de $50 \mathrm{mg} / \mathrm{kg}$ equivale a 
una dosis en humano de $4.1 \mathrm{mg} / \mathrm{kg}$. Por lo tanto considerando las dosis máximas en humanos mencionadas se han usado dosis que son 12 y 16 veces superiores a las recomendadas para piroxicam y meloxicam, respectivamente. Lo que confiere un margen de seguridad en cuanto a la estimación del riesgo genotóxico de estos productos al extrapolar en humanos.

Tabla 1: Frecuencia de eritrocitos policromáticos micronucleados (EPCMN) en células de médula ósea de ratones Swiss a las 24 hs posteriores a la administración ( $\mathrm{F}^{1}$ de Fisher calculado usando ANOVA de un factor; ${ }^{b} \mathrm{p}<0.05$ resultante de aplicar el método de Neuman-Keuls)

\begin{tabular}{|c|c|c|c|c|}
\hline $\begin{array}{l}\text { Compuesto } \\
\text { (NR-CAS) }\end{array}$ & $\begin{array}{c}\text { Dosis } \\
(\mathrm{mg} / \mathrm{kg})\end{array}$ & $\begin{array}{c}\text { EPCMN }{ }^{2} / 24 \mathrm{hs} \\
\text { Datos } \\
\text { individuales }\end{array}$ & $\begin{array}{c}\text { EPCMN/1000EPC } \\
\text { (promedio } \pm \text { DE) }\end{array}$ & $\begin{array}{c}F^{1} \\
\text { (observado) }\end{array}$ \\
\hline \multirow{7}{*}{$\begin{array}{c}\text { Piroxicam } \\
(36322-90-4)\end{array}$} & 0.0 & $3,3,2,4,2,4,4,2$ & $3.00 \pm 0.33$ & \multirow{7}{*}{$\begin{array}{l}2.416(p= \\
0.67)\end{array}$} \\
\hline & 0.3 & $4,1,4,1,5,2,3,2$ & $2.75 \pm 0.53$ & \\
\hline & 1.0 & $4,3,3,2,4,4,3,2$ & $3.12 \pm 0.30$ & \\
\hline & 5.0 & $4,5,4,4,3,2,4,5$ & $3.88 \pm 0.35$ & \\
\hline & 10.0 & $3,1,1,2,4,3,2,2$ & $2.25 \pm 0.37$ & \\
\hline & 0.0 & $2,0,1,3,3,0,1,2$ & $1.50 \pm 0.65$ & \\
\hline & 50.0 & $2,3,2,0,3,2,0$ & $1.71 \pm 0.47$ & \\
\hline \multirow{5}{*}{$\begin{array}{c}\text { 2-aminopiridina } \\
(504-29-0)\end{array}$} & 0.0 & $3,3,2,4,1,5,4,2$ & $3.00 \pm 0.46$ & \multirow{5}{*}{$\begin{array}{l}0.793(p= \\
0.54)\end{array}$} \\
\hline & 0.1 & $3,3,4,2,2,3,1$ & $2.57 \pm 0.37$ & \\
\hline & 0.3 & $4,2,3,2,3,2,2,2,3$ & $2.62 \pm 0.26$ & \\
\hline & 1.0 & $2,3,4,4,6,2,2,3$ & $3.25 \pm 0.49$ & \\
\hline & 5.0 & $2,4,4,3,5,4,3,2$ & $3.38 \pm 0.38$ & \\
\hline \multirow{5}{*}{$\begin{array}{l}\text { Meloxicam } \\
(71125-38-7)\end{array}$} & 0.0 & $1,2,4,2,3,4,1,3$ & $2.50 \pm 0.42$ & \multirow{5}{*}{$\begin{array}{l}1.826(p= \\
0.14)\end{array}$} \\
\hline & 0.1 & $5,3,3,3,5,3,2,4$ & $3.50 \pm 0.38$ & \\
\hline & 5.0 & $6,5,2,5,4,3,2$ & $3.86 \pm 0.59$ & \\
\hline & 10.0 & $2,2,5,4,3,3,2,3$ & $3.00 \pm 0.38$ & \\
\hline & 50.0 & $1,3,3,2,3,4,2,3$ & $2.63 \pm 0.32$ & \\
\hline \multirow{5}{*}{$\begin{array}{c}\text { 2-amino-5-metil } \\
\text { tiazol } \\
(7305-71-7) \\
\end{array}$} & 0.0 & $4,1,2,3,3,5,4,2$ & $3.00 \pm 0.44$ & \multirow{5}{*}{$\begin{array}{l}0.762(p= \\
0.51)\end{array}$} \\
\hline & 0.1 & $3,2,2,2,2,3,3,4,2$ & $2.61 \pm 0.32$ & \\
\hline & 0.3 & $4,2,2,3,3,1,3$ & $2.58 \pm 0.24$ & \\
\hline & 1.0 & $5,4,3,2,3,4,4,2$ & $3.32 \pm 0.36$ & \\
\hline & 5.0 & $4,4,2,3,2,2,6,3$ & $3.22 \pm 0.47$ & \\
\hline \multirow{7}{*}{$\begin{array}{c}\text { Anilina } \\
(62-53-3)\end{array}$} & 0.0 & $3,1,2,2,3,2$ & $2.17 \pm 0.31$ & \multirow{7}{*}{$\begin{array}{l}33.68 \\
(p<0.0001)\end{array}$} \\
\hline & 5.0 & $4,3,0,3,2,4,1,2$ & $2.38 \pm 0.49$ & \\
\hline & 0.0 & $1,2,4,3,3,2,4,1,3$ & $2.56 \pm 0.38$ & \\
\hline & 50.0 & $6,7,5,6,5,7,6$ & $6.00 \pm 0.31^{b}$ & \\
\hline & 100.0 & $5,8,9,7,8,8$ & $7.50 \pm 0.56^{b}$ & \\
\hline & 0.0 & $\begin{array}{l}3,2,5,1,4,2,3,3,4, \\
5\end{array}$ & $3.20 \pm 0.42$ & \\
\hline & 200.0 & $\begin{array}{l}9,6,8,9,4,8,6,9,8, \\
4 \\
\end{array}$ & $7.10 \pm 0.62^{b}$ & \\
\hline
\end{tabular}

\section{CONCLUSIONES}

Bajo las condiciones experimentales utilizadas en el presente estudio los resultados obtenidos indican que los compuestos evaluados no inducen un incremento de eritrocitos policromáticos micronucleados en la médula ósea de los animales tratados en comparación con los controles. Por lo que se consideran como no genotóxicos en las condiciones de estudio utilizadas.

\section{AGRADECIMIENTOS}

Se agradece la colaboración de los docentes de la Cátedra de Química medicinal: Prof. Elsa Gomez, Farm. Estela Ferrato, Farm. Nora Cáceres, Farm. Marcela Garrot y Farm. Rosana Rodriguez. 


\section{REFERENCIAS}

Ashby, J., D.A. Vlachos y H. Tinwell; Activity of aniline in the mouse bone marrow micronucleus assay. Mut. Res.: 263, 115-117 (1991).

Bianchi, M. y A. Panerai; Effects of lornoxicam, piroxicam and meloxicam in a model of thermal hidpaw hyperalgesia induced by formalin inyection in rat tail. Pharm. Res.: 45(2), 101-105 (2002).

Blobaum, A.L. y L.J. Marnett; Structural and functional basis of cyclooxygenase inhibition, J. Med. Chem.: 50(7), 1425-1441 (2007).

Dequeker, J. y otros 10 autores; Improvement in gastrointestinal tolerability of the selective cyclooxygenase (COX)-2 inhibitor, meloxicam, compared with piroxicam: results of the Safety and Efficacy Large-scale Evaluation of COX-inhibiting Therapies (SELECT) trial in osteoarthritis. The British Journal of Rheumatology: 37, 946-951 (1998).

Griswold, D.E. y J.L. Adams; Constitutive Cyclooxygenase (COX-1) and inducible cyclooxygenase (COX-2): rationale for selective inhibition and progress to date. Med. Res. Rev.: 16, 181-206 (1999).

Hayashi, M., T. Sofuni y M. Ishidate Jr.; A pilot experiment for the micronucleus test. The multisampling at multidose levels method, Mutat. Res.: 141, 165-169 (1984).

Lazer, E.S. y otros diecinueve autores; Effect of structural modification of enol-carboxamide-type nonsteroidal antiinflammatory drugs on COX-2/COX-1 selectivity. J. Med. Chem.: 40, 980-989 (1997).

Lipscomb, G.R, N. Wallis, G. Amstrong y D.W. Rees; Gastrointestinal tolerability of meloxicam and piroxicam: a double-blind placebo-controlled study, Br. J. Clin. Pharmacol.: 46(2), 133-137 (1998).

Lombardino, J.G, E.H. Wiserman, W.M. McLamore; Sinthesis and antiinflammatory activity of some 3-carboxamides of 2-alkyl-4-hydroxy-2H-1,2-benzothiazine 1,1-dioxide, J. Med. Chem.: 14, 11711174 (1971).

Mac Gregor, J.T. y otros ocho autores; Guidelines for the conduct of micronucleus assays in mammalian bone marrow erythrocytes, Mutat. Res.: 189, 103-112 (1987).

Praveen Rao, P.N., M.D. Jashin y E.E. Knaus; Design, synthesis and structure activity relationships studies of 3,4,6 triphenylpiran-2-onas as selective cyclooxygenase-2 inhibitors, J. Med. Chem.: 47, 3972-3990 (2004).

Schmid, W.; The micronucleus Test, Mutat. Res.: 31, 9-15 (1975).

Sicardi, S.M. y J.L. Martiarena; Mutagenic and analgesic activities of aniline derivatives, J. Pharm. Sci.: 16, 9-15 (1986a).

Sicardi, S.M. y J.L. Martiarena; Structure-mutagenicity relationships of aromatic amines, IX International Symposiun on Medicinal Chemistry, 54, Berlín, Alemania, 14 a 18septiembre. (1986b).

Sicardi, S.M., J.L. Martiarena y M.T. Iglesias; Mutagenic and analgesic activities of aniline derivatives, J. Pharm. Sci.: 80, 761-764 (1991).

Sicardi, S.M. y E. Ferrato; Mutagenic activity of isoxazolylnaphthoquinoneimines assayed by micronucleus bone marrow test, Mutat. Res.: 343, 61-66 (1995).

Tejer, C.D. y H.C. Doval; La catástrofe del rofecoxib y sus consecuencias inmediatas: la elección de un antiinflamatorio en pacientes con riesgo cardiovascular y una nueva etapa en la introducción de fármacos al mercado, Revista Argentina de cardiología: 72(6), 463-466 (2004). 